\title{
Factores pronósticos y estratificación del riesgo en pacientes con tromboembolia de pulmón aguda sintomática
}

\section{Prognostic Factors and Risk Stratification in Symptomatic Acute Pulmonary Thromboembolism}

\author{
David Jiménez ${ }^{1}$, Deisy Barrios ${ }^{2}$ \\ 1,2,Servicio de Neumología, Hospital Ramón y Cajal, IRYCIS. Madrid \\ ${ }^{1}$ Departamento de Medicina, Universidad de Alcalá de Henares. Madrid
}

La tromboembolia de pulmón (TEP) constituye una enfermedad con un espectro amplio de manifestaciones clínicas, con diferente pronóstico y tratamiento ${ }^{1}$. El tratamiento de elección del grupo de pacientes inestables hemodinámicamente es la fibrinolisis sistémica². Sin embargo, la mayoría de los pacientes con TEP se presentan con pocos síntomas. El tratamiento consiste en el uso de heparina de bajo peso molecular (HBPM), heparina no fraccionada (HNF), o fondaparinux como puente para la anticoagulación oral; 0 de rivaroxaban en monoterapia. La mortalidad precoz de los pacientes estables hemodinámicamente con diagnóstico de TEP oscila entre un 2 y un 10\%. La estratificación de riesgo de los pacientes normotensos con TEP debería usarse para identificar un subgrupo de pacientes de bajo riesgo de mortalidad por todas las causas, que se podrían beneficiar de alta precoz 0 incluso de tratamiento ambulatorio de su enfermedad; y de un subgrupo de pacientes de mayor riesgo de complicaciones asociadas a la propia TEP (TEP de riesgo intermedio; antigua TEP submasiva), que se podrían beneficiar de tratamientos agresivos para su enfermedad (monitorización intensiva, fibrinolisis).

Para la estratificación pronóstica de los pacientes con TEP aguda sintomática se deberían considerar los siguientes principios:

- Para seleccionar pacientes de bajo riesgo subsidiarios de alta precoz o tratamiento ambulatorio, se deberían emplear herramientas con alto valor predictivo negativo de muerte por cualquier causa, recurrencia tromboembólica y sangrados mayores.

- Para seleccionar a pacientes estables hemodinámicamente en los que la trombolisis pudiera ser beneficiosa, se debería usar una herramienta o combinaciones de herramientas con alto valor predictivo positivo para muerte por la propia TEP.

\section{Factores pronósticos}

Los factores pronósticos más habitualmente usados en los pacientes normotensos con TEP se resumen en la Tabla 1. De manera general, estas herramientas evalúan la situación clínica y el estado de salud general del paciente, la disfunción del ventrículo derecho, la carga trombótica y el daño miocárdico.
Tabla 1. Herramientas pronósticas para pacientes normotensos con TEP aguda sintomática

\begin{tabular}{|c|c|}
\hline Marcadores clínicos & $\begin{array}{l}\text { Escala PESI }^{3} \\
\text { Escala PESI simplificada }^{4} \\
\text { Hiponatremia }^{11}\end{array}$ \\
\hline $\begin{array}{l}\text { Marcadores de disfunción del } \\
\text { ventrículo derecho }\end{array}$ & $\begin{array}{l}\text { Ecocardiografía transtorácica }{ }^{12} \\
\text { angioTC }{ }^{12} \\
\text { BNP o NT-proBNP } 12\end{array}$ \\
\hline $\begin{array}{l}\text { Marcadores de carga } \\
\text { trombótica }\end{array}$ & $\begin{array}{l}\text { TVP7 } \\
\text { Dímero D13 }\end{array}$ \\
\hline $\begin{array}{l}\text { Marcadores de daño } \\
\text { miocárdico }\end{array}$ & $\begin{array}{l}\text { cTnl o cTnT'14 } \\
\text { hsTnT }^{6} \\
\text { HFABP }\end{array}$ \\
\hline
\end{tabular}

Abreviaturas: TEP, tromboembolia de pulmón; PESI, Pulmonary Embolism Severity Index; BNP, péptido natriurético cerebral; TVP, trombosis venosa profunda; cTnl, troponina cardiaca I; cTnT, troponina cardiaca T; hsTnT, troponina T de alta sensibilidad; HFABP, proteína ligadora de ácidos grasos cardiacos.

\section{Pacientes de riesgo bajo}

Aujesky et a $\left.\right|^{3}$ derivaron un modelo pronóstico clínico [Pulmonary Embolism Severity Index (PESI)] en 10,354 pacientes ingresados con el diagnóstico de TEP en 186 hospitales de Pensilvania. El modelo incluye 11 parámetros clínicos en el momento de la presentación de los síntomas: edad, sexo, cáncer, insuficiencia cardiaca, enfermedad pulmonar crónica, frecuencia cardiaca $\geq 110 /$ minuto, presión arterial sistólica $<100 \mathrm{~mm} \mathrm{Hg}$, frecuencia respiratoria $\geq 30 /$ minuto, temperatura $<36^{\circ} \mathrm{C}$, estado mental alterado y saturación de oxígeno $<90 \%$. En la serie original, el $2 \%$ de los pacientes pertenecientes a las clases I y || (muy bajo y bajo riesgo) murieron, comparado con el $14 \%$ de las clases III-V (intermedio a muy alto riesgo) en los 30 días posteriores al diagnóstico de la TEP.

Jiménez et al derivaron y validaron una simplificación de la escala PESI (PESIs) ${ }^{4}$. En una cohorte de 995 pacientes, los autores encontraron 6 variables que se asociaron de forma independiente a la mortalidad a 30 días: edad, cáncer, enfermedad cardiopulmonar crónica, taquicardia (frecuencia cardiaca $\geq 110 /$ minuto), hipoxemia (saturación de oxígeno $<90 \%$ ), e hipotensión (presión arterial sistólica < 100 mm $\mathrm{Hg}$ ). La presencia de cualquiera de estas variables incluye a los pacientes en el grupo de alto riesgo. Esta escala fue 
validada por los mismos autores en una cohorte de 7,106 pacientes con TEP del registro RIETE. La escala clasificó al $36.1 \%$ de los pacientes como de bajo riesgo y la mortalidad a 30 días fue de 1.1\% (intervalo confianza [IC] 95\%, 0.7 a $1.5 \%)$.

Recientemente se publicó el ensayo clínico OTPE (Outpatient Treatment of Pulmonary Embolism) (NCT00425542), que evaluó la eficacia y seguridad del tratamiento ambulatorio de la TEP en 344 pacientes de bajo riesgo según la escala PESI (clases de riesgo I y II). En este ensayo, el tratamiento ambulatorio no fue inferior al tratamiento hospitalario convencional en términos de mortalidad, recurrencias trombóticas o sangrados mayores durante las 2 primeras semanas de seguimiento.

En resumen, los modelos clínicos pronósticos son útiles para la selección de pacientes con bajo riesgo de complicaciones en los que se puede considerar el alta precoz 0 el tratamiento ambulatorio. No ha sido completamente aclarado el valor de la combinación de las escalas clínicas y los marcadores bioquímicos (particularmente el péptido natriurético cerebral [BNP] o la troponina de alta sensibilidad [hsTnT]) para la identificación de este grupo de pacientes de bajo riesgo ${ }^{6}$.

\section{Pacientes de riesgo intermedio}

Jiménez et al estudiaron 707 pacientes con un primer episodio de TEP a los que realizaron ecografía completa de miembros inferiores en las 48 horas posteriores al diagnóstico de TEP7 . Los autores encontraron que los pacientes con TEP y trombosis venosa profunda (TVP) presentaron un riesgo significativamente mayor de recurrencia tromboembólica, muerte por cualquier causa y muerte por TEP que Ios pacientes sin TVP. Estos resultados (confirmados en el registro RIETE) apoyan la hipótesis de que el pronóstico de los pacientes con TEP es el resultado de la combinación de la carga trombótica y de la reserva cardiopulmonar. AqueIlos pacientes con peor reserva cardiopulmonar (determinada por pruebas de imagen [ecocardiografía transtorácica 0 angioTC de tórax] o por marcadores biológicos) y/o mayor carga trombótica (determinada por dímero D o ecografía de miembros inferiores) tolerarían peor la rotura del trombo y su embolización al lecho vascular pulmonar. Por tanto, para seleccionar pacientes estables con TEP en los que la fibrinolisis pudiera ser eficaz parece atractiva la combinación de marcadores cardiacos y marcadores de carga trombótica. En un estudio reciente, la combinación de una determinación positiva de troponina cardiaca, la disfunción del ventrículo derecho (determinada por ecocardiografía transtorácica) y el diagnóstico de TVP aumentó el valor predictivo positivo para la muerte por TEP a un $21 \%$.

Se ha finalizado recientemente el reclutamiento del ensayo clínico PEITHO, que pretende evaluar la eficacia y seguridad de tenecteplasa administrada a pacientes normotensos con TEP, que presenten disfunción de ventrículo derecho (por ecocardiografía) y daño miocárdico (elevación de troponina) (NCT00639743).

\section{Pacientes de riesgo alto}

A pesar de que la inestabilidad hemodinámica (definida como shock cardiogénico, tensión arterial sistólica menor de 90 mm Hg o caídas de la tensión arterial sistólica de 40 $\mathrm{mm} \mathrm{Hg}$ no debidas a sepsis, hipovolemia 0 arritmias cardiacas) se ha considerado tradicionalmente una indicación absoluta para la fibrinolisis de los pacientes con TEP ${ }^{2,9}$, el valor predictivo positivo de la hipotensión para mortalidad por la propia TEP sólo fue de un 14\% en el registro MAPETT ${ }^{10}$. Aún más, un estudio reciente realizado en 15,944 pacientes incluidos en el registro RIETE con diagnóstico objetivo de TEP no logró demostrar que la fibrinolisis disminuyera la mortalidad de estos pacientes, ni siquiera en el subgrupo de los pacientes con hipotensión arterial. Por tanto, parece que se deben buscar subgrupos de pacientes hipotensos con TEP en los que el beneficio de la reperfusión precoz supere el riesgo de sangrado asociado al tratamiento trombolítico.

\section{Conclusiones}

Todos los especialistas implicados en el manejo de los pacientes con TEP deberían conocer los métodos de estratificación de riesgo para estos pacientes. La estratificación pronóstica de los pacientes estables con TEP aguda sintomática tiene implicaciones importantes para su manejo y puede contribuir a mejorar el pronóstico de la enfermedad.

\section{Bibliografía}

1. Goldhaber SZ, Visani L, De Rosa M. Acute pulmonary embolism: clinical outcomes in the International Coopeative Pulmonary Embolism Registry. Lancet 1999; 353: 1386-1389.

2. Kearon C, Kahn SR, Agnelli G, et al. Antithrombotic therapy for venous thromboembolic disease: American College of Chest Physicians Evidence-Based Clinical Practice Guidelines (8th edition). Chest 2008; 133: 454-545.

3. Aujesky D, Obrosky DS, Stone RA, et al. Derivation and validation of a prognostic model for pulmonary embolism. Am J Respir Crit Care Med 2005; 172: 1041-1046.

4. Jiménez D, Aujesky D, Moores L, Gomez V, Lobo JL, Uresandi F, Otero R, Monreal M, Muriel A, Yusen RD. Simplification of the Pulmonary Embolism Severity Index for prognosticating patients with acute symptomatic pulmonary embolism. Arch Intern Med 2010; 170: 1383-1389.

5. Aujesky D, Roy PM, Verschuren F, Righini M, Osterwalder J, Egloff M, Renaud B, Verhamme P, Stone RA, Legall C, Sanchez O, Pugh NA, N'gako A, Cornuz J, Hugli 0 , Beer HJ, Perrier A, Fine MJ, Yealy DM. Outpatient versus inpatient treatment for patients with acute pulmonary embolism: an international, open-label, randomised, non-inferiority trial. Lancet 2011; 378: 41-48.

6. Lankeit M, Friesen D, Aschoff J, Dellas C, Hasenfuss G, Katus H, Konstantinides S, Giannitsis E. Highly sensitive troponin T assay in normotensive patients with acute pulmonary embolism. Eur Heart J 2010; 31: 1836-1844.

7. Jiménez D, Aujesky D, Díaz G, Monreal M, Otero R, Martí D, Marín E, Aracil E, Sueiro $A$, Yusen $R$, and the RIETE investigators. Prognostic significance of concomitant deep vein thrombosis in patients presenting with acute symptomatic pulmonary embolism. Am J Respir Crit Care Med 2010; 181: 983-991.

8. Jiménez D, Aujesky D, Moores L, Gómez V, Martí D, Briongos S, Monreal M, Barrios $\mathrm{V}$, Konstantinides S, Yusen RD. Combinations of prognostic tools for identification of high-risk normotensive patients with acute symptomatic pulmonary embolism. Thorax 2011; 66: 75-81.

9. Torbicki A, Perrier A, Konstantinides S, Agnelli G, Galiè N, Pruszczyk P, Bengel F, Brady AJ, Ferreira D, Janssens U, Klepetko W, Mayer E, Remy-Jardin M, Bassand JP, Vahanian A, Camm J, De Caterina R, Dean V, Dickstein K, Filippatos G, Funck-Bren- 
tano C, Hellemans I, Kristensen SD, McGregor K, Sechtem U, Silber S, Tendera M, Widimsky P, Zamorano JL, Andreotti F, Ascherman M, Athanassopoulos G, De Sutter J, Fitzmaurice D, Forster T, Heras M, Jondeau G, Kjeldsen K, Knuuti J, Lang I, Lenzen M, Lopez-Sendon J, Nihoyannopoulos P, Perez Isla L, Schwehr U, Torraca L, Vachiery $\mathrm{JL}$; Task Force for the Diagnosis and Management of Acute Pulmonary Embolism of the European Society of Cardiology. Guidelines on the diagnosis and management of acute pulmonary embolism. Eur Heart J 2008; 29: 2276-2315.

10. Kasper W, Konstantinides S, Geibel A, Olschewski M, Heinrich F, Grosser KD, Rauber $\mathrm{K}$, Iversen S, Redecker M, Kienast J. Management strategies and determinants of outcome in acute major pulmonary embolism: results of a multicenter registry. J Am Coll Cardiol 1997; 30: 1165-1171.

11. Scherz N, Labarère J, Méan M, Ibrahim SA, Fine MJ, Aujesky D. Prognostic importance of hyponatremia in patients with acute pulmonary embolism. Am Respir Crit Care Med 2010; 182: 1178-1183.
12. Sanchez O, Trinquart L, Colombet I, Duriex P, Huisman MV, Chatellier G, Meyer G. Prognostic value of right ventricular dysfunction in patients with haemodynamically stable pulmonary embolism: a systematic review. Eur Heart J 2008; 29: 1569-1577.

13. Lobo JL, Zorrilla V, Aizpuru F, Grau E, Jiménez D, Palareti G, Monreal M; RIETE Investigators. D-dimer levels and 15-day outcome in acute pulmonary embolism. Findings from the RIETE Registry. J Thromb Haemost 2009; 7: 1795-1801.

14. Becattini C, Vedovati MC, Agnelli G. Prognostic value of troponins in acute pulmonary embolism: a meta-analysis. Circulation 2007; 116: 427-433.

15. Dellas C, Puls M, Lankeit M, Schäfer K, Cuny M, Berner M, Hasenfuss G, Konstantinides $S$. Elevated heart-type fatty acid-binding protein levels on admission predict an adverse outcome in normotensive patients with acute pulmonary embolism. J Am Coll Cardiol 2010; 55: 2150-2157.

\section{Normas de publicación}

Galicia Clínica es la revista oficial de la Sociedad Gallega de Medicina Interna (SOGAMI). Se publican 4 números al año, simultáneamente en papel y en www.galiciaclinica.info, incluyéndose en el segundo de ellos las comunicaciones enviadas a la correspondiente Reunión Ordinaria de la SOGAMI.

GALICIA CLÍNICA evaluará para su publicación trabajos médicos relacionados preferentemente con la Medicina Interna y sus subespecialidades, y/o con problemas médicos prevalentes en la Comunidad Autónoma de Galicia

Se admitirán para evaluación trabajos en castellano, gallego, inglés y portugués. Para el envío de originales se ha habilitado un formulario en la pagina web www. galiciaclinica.info. El sistema confirmará la entrega y permitirá consultar el estado del manuscrito. No se aceptarán originales enviados por otros métodos.

El comité editorial, eventualmente con la ayuda de revisores externos, evaluará los trabajos enviados decidiendo si procede su publicación, si es necesario realizar correcciones o si se desestima la publicación. Una vez aceptado, se enviarán a autor las pruebas de imprenta para la corrección de posibles erratas.

Los trabajos reunirán los requisitos de uniformidad habituales en revistas biomédicas. Dichos requisitos se pueden consultar en "Uniform Requirements for Manuscripts Submitted to Biomedical Journals: Writing and Editing for Biomedical Publication, Updated April 2010", disponible en http://www.icmje.org. Se recomienda encarecidamente leer en especial la sección "Preparing a Manuscript for Submission to a Biomedical Journal" (http://www.icmje.org/manuscript_1prepare. $\mathrm{html)} \mathrm{y} \mathrm{sequir} \mathrm{fielmente} \mathrm{sus} \mathrm{indicaciones} \mathrm{a} \mathrm{la} \mathrm{hora} \mathrm{de} \mathrm{redactar} \mathrm{el} \mathrm{trabajo} \mathrm{a} \mathrm{enviar.}$ Se recomienda el empleo de los programas más habituales de edición de texto (Ej. Word) tanto para el texto como para las tablas.

Dado que la mayoría de las páginas se imprimen en blanco y negro, se aconseja evitar en tablas y figuras en la medida de lo posible el uso de colores o tramas que no tengan el adecuado contraste para su identificación.

Las figuras o imágenes se enviarán en archivo aparte, como archivo de imagen (jpeg o similar) o como PDF con una resolución de 300 ppp. a tamaño de impresió definitivo.

\section{La revista presenta las siguientes secciones:}

Editoriales

Habitualmente encargados por la dirección de la revista. Su extensión máxima será de 8 páginas de 30 líneas y se admitirá una figura 0 una tabla y quince citas bibliográficas.

El número máximo de firmantes será de dos.

Originales

Trabajos de investigación sobre cualquier aspecto médico. La estructura general de los trabajos será la tradicional: Título: en el idioma original y en inglés, Resumen y Abstract en inglés, Palabras clave y Key words (que deben corresponder a los medical subjets headings -MESH- del Index Medicus), Introducción, Material y métodos, Resultados, Discusión, Bibliografía

La extensión máxima recomendada del texto es de 20 páginas de 30 líneas, a las que se podrán añadir 5 figuras y 5 tablas, y un máximo de 30 citas bibliográficas. El número máximo de firmantes será de ocho.

\section{Originales breves}

Trabajos de investigación que por sus características no precisan un mayor espacio. Estructura similar a la de los originales. Su extensión máxima será de 10 páginas de 30 líneas, 3 figuras, 3 tablas y 20 citas bibliográficas.

El número máximo de firmantes será de seis.
Revisiones

Habitualmente encargadas por la dirección de la revista. La extensión máxima recomendada del texto es de 30 páginas de 30 líneas, a las que se podrán añadir 6 figuras y 6 tablas, y un máximo de 50 citas bibliográficas.

El número máximo de firmantes será de tres.

Preguntas clínicas

En esta sección se tratará de responder de forma concreta y sucinta a preguntas clínicas concretas que, bien han motivado una controversia, o cuestionan actitudes arraigadas en la práctica diaria. La extensión máxima será de 6 páginas de 30 líneas dos figuras y dos tablas y 15 citas bibliográficas.

El número máximo de firmantes será de dos.

Notas clínicas

Descripción de casos clínicos de excepcional interés. Constarán de una breve introducción, caso clínico, y discusión correspondiente. Su extensión máxima será de 6 páginas, 2 figuras y dos tablas y 15 citas bibliográficas.

El número máximo de firmantes será de cuatro.

\section{Cartas al director}

Comentarios, opiniones u observaciones sobre los diversos trabajos publicados con anterioridad en la revista. La extensión máxima será de 4 páginas de 30 líneas y se admitirá una figura 0 una tabla y diez citas bibliográficas.

El número máximo de firmantes será de dos.

\section{Imágenes médicas}

Imágenes curiosas, insólitas o demostrativas. Se acompañarán con un texto breve como máximo 1 página de 30 líneas, en el que se explique el caso clínico, con una breve discusión acerca de la importancia de la imagen.

El número máximo de firmantes será de dos.

Resúmenes de Tesis doctorales

Elaborados por el autor, describirán el trabajo realizado; su extensión máxima será de 2 páginas de 30 líneas. Debe incluirse un apéndice con los datos correspondientes a Universidad, departamento, director de la tesis y fecha de presentación.

Otros

La dirección de la revista considerará para su publicación cualquier artículo relacionado con la medicina en cualquier aspecto, aunque no se incluya exactamente dentro de los supuestos anteriores. En este caso se recomienda antes de su envío contactar con la dirección para acordar las características del mismo.

En el caso de que los trabajos enviados incluyan imágenes, figuras, tablas o textos sometidos a copyright, será responsabilidad de los autores la obtención de los permisos necesarios para su publicación.

Todas las opiniones 0 afirmaciones expresadas en los artículos corresponden a los autores de los mismos. Tanto el comité editorial como la SOGAMI declinan cualquie responsabilidad a este respecto.

Los trabajos publicados serán propiedad de GALICIA CLÍNICA, cediendo los autores todos los derechos a la misma. 